\title{
A Game Theoretic Model For Cellular Network Operators' Cooperation Under Government Intervention
}

\author{
Atieh Fander \\ Iran University of Science and Technology \\ Saeed Yaghoubi ( $\nabla$ yaghoubi@iust.ac.ir) \\ Iran University of Science and Technology https://orcid.org/0000-0003-1218-9050 \\ Javad Tajik \\ Iran University of Science and Technology
}

\section{Research Article}

Keywords: Game theory, Cellular network Operators, Government intervention, Cellular network operators, Cellular antennas, Coverage development rate

Posted Date: August 9th, 2021

DOI: https://doi.org/10.21203/rs.3.rs-592939/v1

License: (c) (i) This work is licensed under a Creative Commons Attribution 4.0 International License. Read Full License

Version of Record: A version of this preprint was published at RAIRO - Operations Research on February 10th, 2022. See the published version at https://doi.org/10.1051/ro/2022025. 


\title{
A game theoretic model for cellular network operators' cooperation under government intervention
}

\author{
Atieh Fander ${ }^{\mathrm{a}}$, Saeed Yaghoubi ${ }^{\mathrm{a}, 1}$, Javad Tajik \\ ${ }^{a}$ School of Industrial Engineering, Iran University of Science and Technology, Tehran, Iran
}

\begin{abstract}
In the digital world today, cellular networks and their operators play a competitive and important role in communications. The basis of the competition of operators, the quality of provided services, and the coverage level of their antennas, thereby attract customers. This paper studies cellular networks with two old and new operators and under the influence of government intervention in one area. Due to the high cost of building an antenna, the new operator participates in the cost of the infrastructure of the old operator to use the services of these antennas for their customers. On the other hand, the government considers incentive schemes to support mobile operators. The government plan is that he takes part in the infrastructure cost of the old operator and will receive it an income tax; to support the new operator declare it exempt from tax. The government subsidy contract with the old operator is based on the coverage level of the antenna and supports the operator to increase the coverage level. Some numerical examples for Iranian telecommunication companies are applied to examine the applicability of the proposed models. Finally, sensitivity analysis on the main parameters is analyzed in-depth to extract some managerial implications.
\end{abstract}

Keywords: Game theory; Cellular network Operators; Government intervention; Cellular network operators; Cellular antennas; Coverage development rate.

\section{Introduction}

In the past decade, the development of information and communication has brought about many changes in human life by providing services such as multimedia communications, highquality videos, voice and video calls, internet, and so on. This rapid growth in communications is due to the increase in mobile phone users, so that, regardless of income and lifestyle, smartphones have become an essential part of people in daily life. Smartphones are equipped with a receiver and transmitter system that transmits data to the cellular network via this interface. The popularity and speed of access to services provided by cellular networks have made them one of the most powerful players in communications. For this reason, in 2017, Global System for Mobile Communications or GSM Association reports that more than half of the world's population uses mobile services (Berrocal-Plaza, Vega-

${ }^{1}$ Corresponding Author: School of Industrial Engineering, Iran University of Science and Technology, Narmak, Tehran, Iran Postal code: 16846-13114, Tell: +982173225053; Fax: +982173225098, E-mail Address: yaghoubi@iust.ac.ir 
Rodríguez, \& Sánchez-Pérez, 2015). This has created a technological and economic competitive environment between cellular networks operators, each of which seeks to provide better quality services and subsequently attract more demand. A higher level of quality, better services, and lower energy consumption indicate the performance of the cellular network, so managers are looking for a way to optimize network design. The infrastructure and antennas of any mobile network are owned by an operator.

A cellular network operator is a telecommunications service provider that provides voice and data-dependent services to users. Therefore, cellular network operators design a variety of mobile networks and offer different services, based on geographical conditions and customer preferences, in order to attract more demands in wider coverage. Bandwidth traffic is one of the parameters that plays an important role in customer dissatisfaction and the imposition of economic costs on operators, which is why operators are seeking to increase the quality of communications and reduce traffic volumes. The data and information have shown that messages, when transmitted to mobile users, occupy more than $33 \%$ of the signaling traffic passing through the bandwidth (Berrocal-Plaza, Vega-Rodríguez, \& SánchezPérez, 2014). In the first generation (1G) cellular system had a smaller bandwidth so that supported $10 \mathrm{Kbps}$ data rates and the second $(2 \mathrm{G})$ and third (3G) generations were produced with higher quality. However, through the evolution of wireless networks, the fourth generation (4G) has been released with the highest quality data transmission and peak data rates reached about 1 Gbps (Xifilidis \& Psannis, 2018).Thus, the quality and coverage have become competitive advantages for operators.

In recent years, due to the growing number of mobile operators, a competitive environment has been created between operators to absorb more demand and subsequently higher profits. For this reason, operators are trying to create an antenna coverage for their users in all geographic areas so that they cope with demand satisfaction. On the other hand, operators provide two types of SIM cards to answer all customers, one of which is a prepaid card and other SIM cards are permanent. The price of prepaid SIM card is lower than the permanent SIM card and requires a periodic charge, but it costs more for services. Occasionally, operators are constructing jointly a cellular antennas network due to reduced infrastructure costs, with the goal of meeting the demand of their customers (Chan \& McNaught, 2008). The government subsidizes operators for the purpose of customer satisfaction and the raising of the quality and coverage of the mobile network; on the other hand, it receives tax on operators' income.

In this paper, a model is developed for cellular networks consisting of an old operator, a new operator, and a government with four kinds of deterministic demand. The demand for operators is divided into two parts, which the first part is the demand for prepaid SIM cards and the second part is the demand for permanent SIM cards. The demand for operator $A$ is affected by quality level and coverage development rate which are two endogenous 
variables. Similarly, the demand for operator $B$ is affected by permanent SIM card's price and prepaid SIM card's price which are two endogenous variables. Because operator $A$ is present in this area and provides services, the operator $A$ 's SIM cards prices are specified as parameters. Also, because operator $A$ is older and the antennas are in its possession, the quality level is determined by operator $A$ and the operator $B$ does not interfere. The main contributions of the current paper that distinguish it from the other similar investigations are stated below.

- Presenting a novel mathematical model for cellular networks members' cooperation with the view of government intervention.

- Analyzing the effect of coverage development rate and quality level in cellular networks due to their impacts on demand.

- Implementing a cooperation agreement between two operators for the participation of the operator $B$ in the infrastructure cost and use the services.

- Proposing a novel development rate based subsidies contract as the government tool to encourage the operator $A$.

It is noteworthy to mention that in a few articles in the literature, the relationship between cellular networks has considered as a game. One of this articles is presented by Srivastava et al. (2005) and the other one is investigated by Kamal, Coupechoux, and Godlewski (2009) but in none of both papers, these relations have not presented as a mathematical model. In the current paper, for the first time, relationships between members are examined in the mathematical model, and the difference between the prices of c prepaid and permanent SIM cards as well as the price of their services has been addressed. Also, in the proposed model, the importance of the quality level of services and the coverage rate of the cellular network has been considered. It should be noted that no article in the literature has implemented a coverage development rate subsidiary from the government to encourage operators. The remainder of this paper is organized as follows. Section 2 reviews the relevant literature. In Section 3, the problem description is presented. Section 4 contains the mathematical models of the centralized and decentralized system. In Section 5, a novel coverage level based contract is suggested to coordinate the operator $A$ and government. In Section 6, some sensitivity analysis is conducted to illustrate the applicability of the proposed models. Concluding remarks are presented in the final section.

\section{Literature Review}

According to studies conducted in the field of cellular networks, it has become clear that most of the papers in this field have been devoted to the design of the cellular network, and few articles have focused on game theory in this issue. Initially, the review of the network design articles has been addressed. Due to the wide range of sensors, wireless networks have attracted many research efforts. In the same way, Cheng, Narahari, Simha, Cheng, and Liu 
(2003) proposed two heuristic methods: power assignment based on minimum spanning tree and incremental power transmission levels to the sensors with aiming at minimizing energy cost. One of the problems with wireless networks is energy consumption in the transmission of information, which depends on the next node's intersection selection. More precisely, this can be called a routing problem. Chang and Tassiulas (2004) considered routing problem as a linear programming problem and, on the other hand, they sought to optimize network lifetime. Zhang and Hou (2005) provided a cover model for wireless networks, with the least number of active sensors providing more coverage and connectives. Given the importance of designing a cellular network in mobile telecommunication systems, Talbi, Cahon, and Melab (2007) proposed a multi-objective model of the problem in its full practical complexity with the goal of improving the quality of networks and their robustness. In order to compute a set of diversified non-dominated networks, the Pareto approach is used in their paper. By using three realistic criteria the efficiency and the reliability of hierarchical parallel hybrid models are shown. Liu, Hu, and Li (2010) maximized network lifetime, coverage, and connectives with applying deployment strategies based on voronoi polygons to increase coverage in wireless mobile sensor networks. Lanza-Gutierrez and Gomez-Pulido (2015) provided a network model for assessing the efficiency deploy energy relay nodes in previously low-cost static wireless sensor networks with the aim of optimizing the average energy cost, sensitivity, and network reliability. Due to the scale and complexity of the model, the metaheuristics algorithms such as the two standard genetic Algorithms, the trajectory algorithm, and two novel swarm intelligence algorithms have been used as a solution method. Salih, Hang See, Ibrahim, Yussof, and Iqbal, A. (2015) developed a noncooperative game-theoretic model and strategy space according to user preference to solve network selection problems. Mohamadi, Yaghoubi, and Pishvaee (2016) developed a fuzzy scenario-based optimization model for telecommunication towers concerning humanitarian relief logistics in which the problem was formulated in a nonlinear and multi-objective model. Keskin (2017) suggested a column generation heuristic approach for optimizing a mixed integer linear programming model of wireless sensor network design with mobile sinks. Also Ekmen and Altın-Kayhan (2017) proposed a novel strategy for multicopy and multi-path routing, regarding to improve energy efficient. Considering reliability concept, they developed a mixed integer programming model. Additionally, Dahi, Alba, and Draa (2018) have proposed a new low-complexity adaptive cellular genetic algorithm for optimizing the mobile phone tracking process, which it has implemented in 25 differentlysized realistic mobile networks and the results have assessed.

Due to the presence of different decision makers in wireless networks, a mathematical analysis in the form of a game will create a competitive and attractive atmosphere among decision makers. However, by the reason of the complexity of mobility and traffic models, the dynamic topology, and the unpredictability of link quality, mathematical analysis of ad- 
hoc wireless networks has not been effective and successful. Srivastava et al. (2005) developed a model that examines the various interactions in these networks as game theory and motivates decision makers to participate in social activities and increase profits. Ji and Liu (2007) investigated a game theoretical overview of dynamic spectrum sharing in an adhoc wireless network from three aspects: analysis of network users' behaviors, efficient dynamic distributed design, and optimality analysis. Kamal et al. (2009) proposed a game theoretical model to analyze the interaction among cellular operators with packet services in cellular networks. For this model, there are three objectives: user productivity, spectrum price, and the blocking probability into consideration. Trestian, Ormond, and Muntean (2012) studied an overview issues and challenges of cellular decision making, a comprehensive classification of related game theoretic approaches, and a discussion on the application of game theory problem that next generation of $4 \mathrm{G}$ deals with them. To improve the energy efficiency of green cellular networks, Zheng et al. (2015) presented a distributed cooperative approach by graphical game, where each base station acts as a game player with the constraint of traffic load. Qin, Wang, Wang, Lin, and Wang (2019) developed a Probabilistic Routing scheme based on Game Theory (PRGT) to stimulate cooperation among selfish nodes. Also they implemented a Markov-based probability prediction model to investigate selecting the rely node. It is worth mentioning that Fander and Yaghoubi (2021b) recently proposed the mathematical models for cooperation among mobile phone manufacturer, mobile application developers, and mobile operators based on a revenue sharing contract and without government intervention.

In the discussion of the game theory in the reviewed articles, the government intervention is shown as a supportive and motivational tool for organizations and customers. Sheu and Chen (2012) investigated the impacts of financial government interventions on the competition of green Supply chains. Their analysis based on a three-stage game model, showed that if the government becomes involved as a facilitator, the profitability of the green supply chains improves dramatically. Hafezalkotob (2015) proposed a model with considering price competition of two supply chains, one green and one regular, under the government financial intervention. He analyzed the impact of government tariffs on the players' optimal policies. Mahmoudi and Rasti-Barzoki (2018) examined the contradiction between government goals and the goals of the manufacturers in the sustainable supply chain for the first time. Three different scenarios for the government are considered. In the first scenario, the government applies taxes and subsidies in order to maximize its profit with the highest overall environmental impact. In the second scenario, the government determines tariffs that calculate the overall environmental impact at least with respect to their lower profit margin. Finally, in the third scenario, the government eliminates the goal of profit and environmental goals through a linear combination in a goal function. In addition, Hafezalkotob (2018) presented twelve mathematical programming models using 
the Stackelberg game between government and supply chain. A comprehensive analysis of the supply chain shows some managerial insights, which have indicated that all interventions are beneficial because they lead to more social services than prohibitive policies. Zand, Yaghoubi, and Sadjadi (2019) analyzed government direct limitation on closed-loop supply chain members' optimal decisions and profit by determining certain thresholds for products greening level based on maximizing government social welfare. Recently, a three-echelon closed-loop solar cell supply chain including a supplier, a thirdparty logistics (3PL), and an assembler is presented by Kharaji Manouchehrabadi and Yaghoubi (2020). Fander and Yaghoubi (2021a) recently analyzed the government supervision in fuel-efficient technology on automotive and fuel supply chain with two policies of subsidy-taxation and granting loans to customers.

In none of the above-mentioned articles, the participation of two operators is considered in the construction of a cellular network with government support. Furthermore, due to the importance of the coverage level in customer satisfaction, the government allocates subsidies to operators on this basis. To the best of our knowledge, this paper is the first study that analyzes the decisions of operators and government besides the improvement of operators profit and government utility.

\section{Problem Description}

First, all of the notations applied in this paper are illustrated in Table 1. Then, consider a cellular network with an old operator $A$ and a new operator $B$, in which operator $B$ has participated in the operator $A$ 's infrastructure costs. In this network, the government supports the operator $A$ with considering subsidies on the infrastructure cost, in order to increase services quality and coverage development rate and on the other hand, receives income tax. It is worth noting that the operator $B$ does not include government subsidiary support and has been exempted by taxes to support this operator. Fig. 1 shows the structure of the cellular network. 


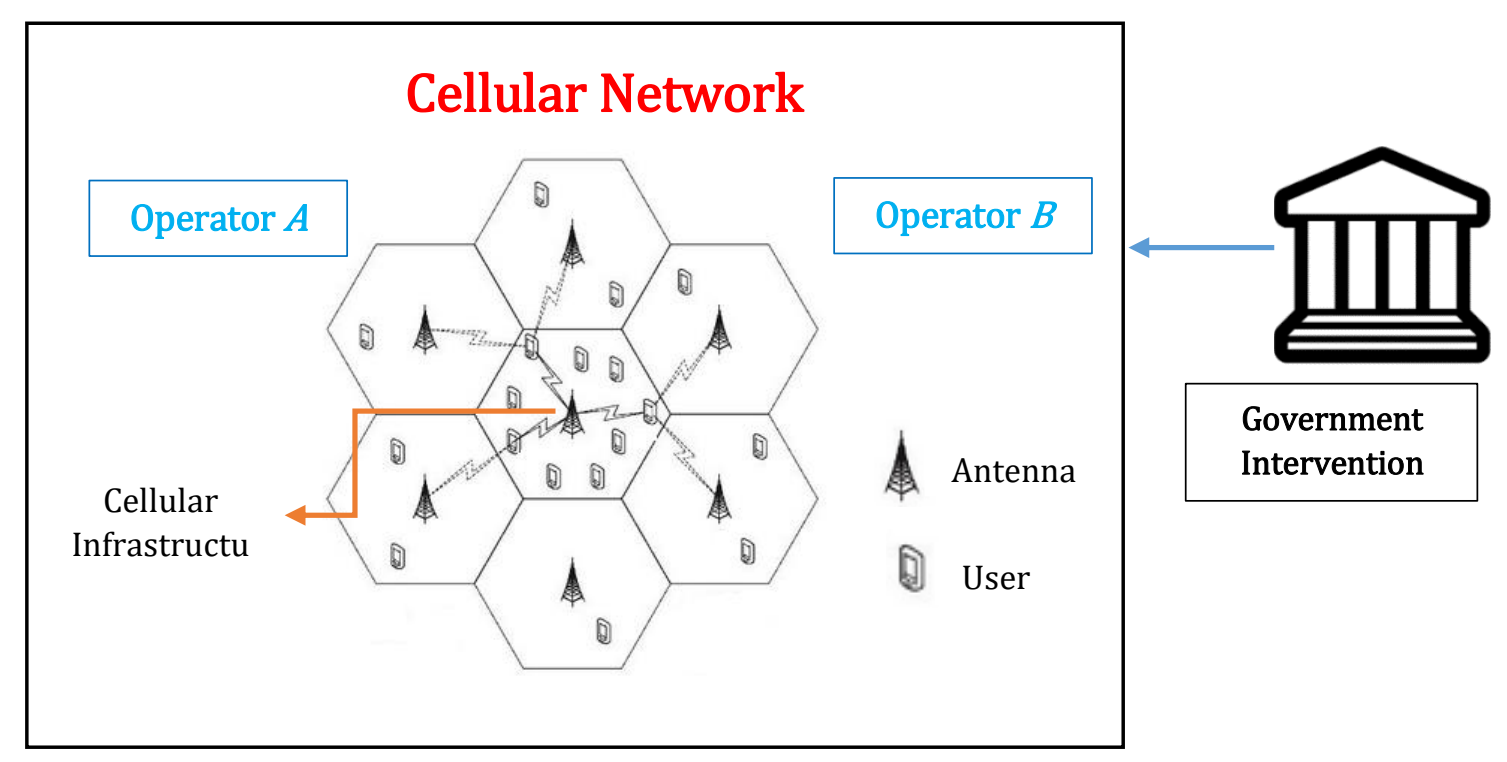

Fig. 1 the structure of the cellular network

Operator $B$ receives a $\varphi$ rate of its customers' demand free from the network due to its participation in costs, and the rest of its demand is purchased from the operator $A$ with a discount price based on the percentage of participation.

Table 1. Parameters and decision variables

\begin{tabular}{cl}
\hline$\alpha$ & Coefficient of demand ratio \\
$r$ & Coverage development rate \\
$a_{1}$ & Potential demand for prepaid SIM cards \\
$a_{2}$ & Potential demand for permanent SIM cards \\
$b$ & Competitive services price coefficient \\
$p^{A, k, 1}$ & The selling price of the prepaid SIM card's services for operator $A$ \\
$p^{B, k, 1}$ & The selling price of the prepaid SIM card's services for operator $B$ \\
$p^{A, k, 2}$ & The selling price of the permanent SIM card's services for operator $A$ \\
$p^{B, k, 2}$ & The selling price of the permanent SIM card's services for operator $B$ \\
$D$ & The demand average \\
$c$ & Competitive SIM cards price coefficient \\
$d$ & Coefficient of quality level \\
$\eta$ & Coefficient of infrastructure rate \\
$\varphi$ & Participation rate of operator $B$ \\
$\gamma$ & Government taxes rate \\
$r_{0}$ & Coefficient of coverage development rate \\
$q$ & Quality level of operator $A$ \\
$m$ & Coefficient of coverage development rate costs \\
$k$ & Coefficient of quality level costs \\
$\lambda_{1}$ & Coefficient of the government's economic satisfaction factor \\
$\lambda_{2}$ & Coefficient of the government's quality level satisfaction factor \\
$\lambda_{3}$ & Coefficient of the government's coverage development satisfaction factor \\
$D^{A, 1}$ & The demand of prepaid SIM card of operator $A$ \\
$D^{A, 2}$ & The demand of permanent SIM card of operator $A$ \\
$D^{B, 1}$ & The demand of prepaid SIM card of operator $B$ \\
\hline
\end{tabular}




\begin{tabular}{cl}
\hline$D^{B, 2}$ & The demand of permanent SIM card of operator $B$ \\
$p^{A, s i m, 1}$ & The price of prepaid SIM card of operator $A$ \\
$p^{A, s i m, 2}$ & The price of permanent SIM card of operator $A$ \\
$p^{B, \text { sim, }, 1}$ & The price of prepaid SIM card of operator $B$ \\
$p^{B, \text { sim, } 2}$ & The price of permanent SIM card of operator $B$ \\
$\Pi_{A}$ & The profit of operator $A$ \\
$\Pi_{B}$ & The profit of operator $B$ \\
$\Pi_{T}$ & The total profit centralized system \\
\hline
\end{tabular}

The main assumptions of this paper are stated as follows:

- Market demand for operator $A$ is a function of its coverage development rate and technology level, which it has two part for permanent and prepaid SIM cards. Let $D^{A, 1}(r, q)=\alpha r r_{0} a_{1}-b\left(p^{A, k, 1}-p^{B, k, 1}\right)-c\left(p^{A, \operatorname{sim}, 1}-p^{B, s i m, 1}\right)+d(q)$ and $D^{A, 2}(r, q)=\alpha r \mathrm{r}_{0} a_{2}-b\left(p^{A, k, 2}-p^{B, k, 2}\right)-c\left(p^{A, \operatorname{sim}, 2}-p^{B, s i m, 2}\right)+d(q)$, where $a>0$, $b>0, c>0, d>0$, and $r_{0}>0$. Note that $D^{A, 1}(r, q), D^{A, 2}(r, q)>0$ is always satisfied.

- Market demand for operator $B$ is a function of its sale prices for prepaid and permanent SIM cards' services, which it has two part for permanent and prepaid SIM cards. Let $D^{B, 1}\left(p^{B, k, 1}\right)=(1-\alpha) r r_{0} a_{1}-b\left(p^{B, k, 1}-p^{A, k, 1}\right)-c\left(p^{B, s i m, 1}-p^{A, \operatorname{sim}, 1}\right)+$ $d(q)$ and $D^{B, 2}\left(p^{B, k, 2}\right)=(1-\alpha) r r_{0} a_{2}-b\left(p^{B, k, 2}-p^{A, k, 2}\right)-c\left(p^{B, s i m, 2}-p^{A, \operatorname{sim}, 2}\right)+$ $d(q)$, where $a>0, b>0, c>0, d>0$, and $r_{0}>0$. Note that $D^{B, 1}\left(p^{B, k, 1}\right), D^{B, 2}\left(p^{B, k, 1}\right)>$ 0 is always satisfied.

- Energy costs are included in the operating cost.

- $\quad$ Operator $B$ does not receive subsidies from the government and is exempt from taxes.

\section{Mathematical Modeling}

According to the problem descriptions, the behavior of the cellular network and the government can be examined as follows. Operator $B$ participates in infrastructure costs of operator $A$ as $\varphi$ rate and uses the services of cellular network. Operator $A$ wants to improve its coverage development rate and quality level to increase its profit and operator $B$ tries to decrease its services price to have more profit. The government intervenes in this network and contributes to infrastructure costs as $r r_{0}$ rate and receives revenue taxes as $\gamma$ rate from operator $A$.

\subsection{Centralized System}

In this case, there is no centralized model between operator $A$ and operator $B$. Due to the point that customers of operators are independent of each other, each of the operators provides the right service to request their customers. The two operators also have different SIM cards and only use a common antenna in this area. If these two operators are integrated, their business goals will deal with many challenges. Both parties are concerned about their own business condition, and they decide to participate with each other to raise profits and reduce costs.

\subsection{Decentralized System}


In this section, the operator $A$ seeks to increase its own profit. Operator $A$ faces a demand, which varies with quality level and coverage development rate. Indeed, as quality level $(q)$ and coverage development rate $(r)$ increase, demand increases; likewise, increase in the demand value of operator $A$ leads to an increase in operator $A$ 's profit. On the other hand, Operator $B$ does not have infrastructure and antenna at this location, but because of the considerable demand, it wants to provide services at this location. Operator $B$ copes with a demand, which varies with prepaid SIM card services price $\left(p^{B, k, 1}\right)$ and permanent SIM card services price $\left(p^{B, k, 2}\right)$. Actually, by increasing prepaid SIM card services price $\left(p^{B, k, 1}\right)$ and permanent SIM card services price $\left(p^{B, k, 2}\right)$, demand decreases; so, increase in the demand value of operator $B$ leads to a decrease in operator $A$ 's profit. Therefore, it is economical for operator $B$ to participate in part of the infrastructure costs of operator A instead of building a new network, thereby keeping prices low and benefiting from services. Operator $B$ uses part of its demand, which is equal to the participation rate $(\varphi)$, to utilize the network free of charge and buys the rest of its demand from the operator $A$ at a discounted price is given as $(1-\varphi) p^{A, k, 1}$. Thus, both parties will achieve their goals; operator $A$ will profit from lower infrastructure costs, thereby improving the quality and level of coverage, and operator $B$ will profit from lower the price of its services and will also get higher profits. In this Stackelberg game, which is a non-cooperative game, operator $A$ is the leader and operator $B$ is the follower. The total profit generated by operator $A$ is as follows:

$$
\begin{aligned}
& \Pi_{A}=\left(p^{A, k, 1} D^{A, 1}(r, q)+p^{A, k, 2} D^{A, 2}(r, q)+p^{A, s i m, 1}\left(\frac{\alpha r a_{1}}{D}\right)+p^{A, s i m, 2}\left(\frac{\alpha r a_{2}}{D}\right)\right)- \\
& (1-\varphi)\left(k q^{2}+m\left(r r_{0}\right)^{2}\right)+(1-\varphi)\left(D^{B, 1}\left(p^{B, k, 1}\right)+D^{B, 2}\left(p^{B, k, 2}\right)\right)(1-\varphi) p^{A, k, 1}- \\
& \boldsymbol{c} \eta\left(D^{A, 1}(r, q)+D^{A, 2}(r, q)+D^{B, 1}\left(p^{B, k, 1}\right)+D^{B, 2}\left(p^{B, k, 2}\right)\right)
\end{aligned}
$$

In Eq. (1), the first and second terms indicate the sales revenue from prepaid and permanent SIM cards services. The third and fourth terms state the sales revenue from prepaid and permanent SIM cards according to the point that ratio of the demand is calculated by dividing the coverage into the average demand, which indicates the number of SIM cards. The fifth term refers to the Infrastructure costs based on the quality level and coverage development rate. The sixth term is sales revenue of operator B's demand. The seventh term concerns to operational and energy costs.

Theorem 4.1. The operator $A$ 's profit function is concave in $r$ and $q$. Thus, the optimal and exclusive $(r, q)$ can be obtained to maximize the operator $A$ 's expected profit. The optimal coverage development rate and quality level are calculated as follows, respectively. 


$$
\begin{gathered}
r=\frac{-2 \alpha\left(a_{1} p^{A, s i m, 1}+a_{2} p^{A, s i m, 2}\right)+\left(2 a_{1} \eta+a_{1}(-2+(-1+\alpha)(-2+\varphi) \varphi) p^{A, k, 1}\right.}{4(-1+\varphi) m r_{0}{ }^{2} D} \\
+\frac{\left.a_{2}\left(2 \eta+(-1+\alpha)(-1+\varphi)^{2} p^{A, k, 1}-(1+\alpha) p^{A, k, 2}\right)\right) r_{0} D}{4(-1+\varphi) m r_{0}{ }^{2} D} \\
q=\frac{d\left(8 \eta+(-5-2(-2+\varphi) \varphi) p^{A, k, 1}-3 p^{A, k, 2}\right)}{4(-1+\varphi) k}
\end{gathered}
$$

Proof. Since the first principal minor is negative $\left(-2(1-\varphi) m r_{0}{ }^{2}<0\right)$ and the second principal minor is positive $\left(4(1-\varphi)^{2} m r_{0}{ }^{2} k>0\right)$, the operator $A$ 's expected profit function is concave in both $r$ and $q$. Therefore, the optimal solution can be achieved if the corresponding parameters satisfy the conditions mentioned for concavity.

$$
\begin{aligned}
& H=\left[\begin{array}{ll}
\frac{\partial^{2} \Pi_{A}}{\partial r^{2}} & \frac{\partial^{2} \Pi_{A}}{\partial r \partial q} \\
\frac{\partial^{2} \Pi_{A}}{\partial r \partial q} & \frac{\partial^{2} \Pi_{A}}{\partial q^{2}}
\end{array}\right] \\
& H=\left[\begin{array}{cc}
-2(1-\varphi) m r_{0}{ }^{2} & 0 \\
0 & -2(1-\varphi) k
\end{array}\right]
\end{aligned}
$$

The profit generated by operator B is as follows:

$$
\begin{aligned}
& \Pi_{B}=\left(p^{B, k, 1} D^{B, 1}\left(p^{B, k, 1}\right)+p^{B, k, 2} D^{B, 2}\left(p^{B, k, 2}\right)+p^{B, \operatorname{sim}, 1}\left(\frac{D^{B, 1}\left(p^{B, k, 1}\right)}{D}\right)+\right. \\
& \left.p^{B, s i m, 2}\left(\frac{D^{B, 2}\left(p^{B, k, 2}\right)}{D}\right)\right)-\varphi\left(k q^{2}+m\left(r \mathrm{r}_{0}\right)^{2}\right)-(1-\varphi)\left(D^{B, 1}\left(p^{B, k, 1}\right)+\right. \\
& \left.D^{B, 2}\left(p^{B, k, 2}\right)\right)(1-\varphi) p^{A, k, 1}
\end{aligned}
$$

In Eq. (4), the first and second terms indicate the sales revenue from prepaid and permanent SIM cards services. The third and fourth term state the sales revenue from prepaid and permanent SIM cards according to the ratio of the demand multiplied by services prices to the average demand, which indicates the number of SIM cards. The fifth term refers to the participation rate cost in infrastructure costs based on the quality level and coverage development rate. The sixth term is purchases cost of the demand.

Theorem 4.2. The operator B's profit function is concave in $p^{B, k, 1}$ and $p^{B, k, 2}$. Thus, the optimal and exclusive $\left(p^{B, k, 1}, p^{B, k, 2}\right)$ can be obtained to maximize operator $B$ s expected profit. The optimal prepaid SIM cards services price and permanent SIM cards services price are calculated as follows, respectively.

$$
\begin{aligned}
& p^{B, k, 1}=\frac{1}{8 b(-1+\varphi) k m r_{0} D}\left(a _ { 1 } ^ { 2 } ( - 1 + \alpha ) k \left(2 \alpha p^{A, s i m, 1}+(-2 \eta+(2-(-1+\alpha)(-2+\right.\right. \\
& \left.\left.\varphi) \varphi) p^{A, k, 1}\right) \mathrm{r}_{0} D\right)+a_{1} a_{2}(-1+\alpha) k\left(2 \alpha p^{A, s i m, 1}+(-2 \eta-(-1+\alpha)(-1+\right.
\end{aligned}
$$




$$
\begin{aligned}
& \left.\left.\varphi)^{2} p^{A, k, 1}+p^{A, k, 2}+\alpha p^{A, k, 2}\right) \mathrm{r}_{0} D\right)+m \mathrm{r}_{0}\left(\left(-d^{2}\left((5+2(-2+\varphi) \varphi) p^{A, k, 1}+\right.\right.\right. \\
& \left.\left.3 p^{A, k, 2}\right)+4 c\left(2 d^{2} \eta+(-1+\varphi) k\left(p^{A, s i m, 1}-p^{B, s i m, 1}\right)\right)\right) D+4 b(-1+ \\
& \left.\left.\left.\mathrm{f} \varphi) k\left(-p^{B, s i m, 1}+(2+(-2+\varphi) \varphi) p^{A, k, 1} D\right)\right)\right)\right) \\
& p^{B, k, 2}=\frac{1}{8 b(-1+\varphi) k m r_{0} D}\left(a _ { 1 } ^ { 2 } ( - 1 + \alpha ) k \left(2 \alpha p^{A, s i m, 1}+\varphi(-\alpha(-2+\varphi)+\right.\right. \\
& \left.\varphi) p^{A, k, 1} \mathrm{r}_{0} D-2\left(\eta+(-1+\varphi) p^{A, k, 1}\right) \mathrm{r}_{0} D\right)+a_{2}{ }^{2}(-1+\alpha) k\left(2 \alpha p^{A, s i m, 2}+\right. \\
& \left.\alpha\left(-(-1+\varphi)^{2} p^{A, k, 1}+p^{A, k, 2}\right) \mathrm{r}_{0} D+\left(-2 \eta+(-1+\varphi)^{2} p^{A, k, 1}+p^{A, k, 2}\right) \mathrm{r}_{0} D\right)+ \\
& m \mathrm{r}_{0}\left(\left(-d^{2}\left((5+2(-2+\varphi) \varphi) p^{A, k, 1}+3 p^{A, k, 2}\right)+4 c\left(2 d^{2} \eta+(-1+\varphi) k\left(p^{A, s i m, 2}-\right.\right.\right.\right. \\
& \left.\left.\left.\left.\left.p^{B, \operatorname{sim}, 2}\right)\right)\right) D+4 b(-1+\varphi) k\left(-p^{A, s i m, 2}+\left((-1+\varphi)^{2} p^{A, k, 1}+p^{A, k, 2}\right) D\right)\right)\right)
\end{aligned}
$$

Proof. Since the first principal minor is negative $(-2 b<0)$ and the second principal minor is positive $\left(4 b^{2}>0\right)$, the operator $B$ s expected profit function is concave in both $p^{B, k, 1}$ and $p^{B, k, 2}$. Therefore, the optimal solution can be achieved if the corresponding parameters satisfy the conditions mentioned for concavity.

$$
\begin{aligned}
& H=\left[\begin{array}{cc}
\frac{\partial^{2} \Pi_{B}}{\partial p^{B, k, 1^{2}}} & \frac{\partial^{2} \Pi_{B}}{\partial p^{B, k, 1} \partial p^{B, k, 2}} \\
\frac{\partial^{2} \Pi_{B}}{\partial p^{B, k, 1} \partial p^{B, k, 2}} & \frac{\partial^{2} \Pi_{B}}{\partial p^{B, k, 2^{2}}}
\end{array}\right] \\
& H=\left[\begin{array}{cc}
-2 b & \mathbf{0} \\
\mathbf{0} & -2 b
\end{array}\right]
\end{aligned}
$$

\section{Government Intervention}

In many cases, the government considers subsidies to support businesses for helping them profitably and reduce costs, and versus receive tax from them. In this case, the government decides to take part in infrastructure costs in order to support the old operator. On the other hand, to encourage the operator $A$ to improve the quality level and increase coverage development rate, the rate of its partnership is based on the increased coverage development rate. The purpose of the government is to support operator $A$ in costs, and from the other side, by increasing the quality level and the coverage development rate, it will enhance customer satisfaction and improve the social responsibility aspects. The government does not receive taxes from operator B to support him and does not subsidize his costs. The government contribution in infrastructure costs is $\left(r \mathrm{r}_{0}\right)$ and the tax rate that receives from operator $A$ is $(\gamma)$. In this Stackelberg model, the government is the leader and operator A and operator B are the followers. The total profit generated by operator $A$ after government support is calculated as follows: 


$$
\begin{aligned}
& \Pi_{A}=(1-\gamma)\left(p^{A, k, 1} D^{A, 1}(r, q)+p^{A, k, 2} D^{A, 2}(r, q)+p^{A, s i m, 1}\left(\frac{\alpha r a_{1}}{D}\right)+\right. \\
& \left.p^{A, \operatorname{sim}, 2}\left(\frac{\alpha r a_{2}}{D}\right)\right)-\left(1-\varphi-r \mathrm{r}_{0}\right)\left(k q^{2}+m\left(r \mathrm{r}_{0}\right)^{2}\right)+(1-\gamma)(1-\varphi)\left(D^{B, 1}\left(p^{B, k, 1}\right)+\right. \\
& \left.D^{B, 2}\left(p^{B, k, 2}\right)\right)(1-\varphi) p^{A, k, 1}-\eta\left(D^{A, 1}(r, q)+D^{A, 2}(r, q)+D^{B, 1}\left(p^{B, k, 1}\right)+\right. \\
& \left.D^{B, 2}\left(p^{B, k, 2}\right)\right)
\end{aligned}
$$

The total utility generated by the government is calculated as follows:

$$
\begin{aligned}
& \Pi_{\boldsymbol{g}}=\lambda_{1} \gamma\left[\begin{array}{c}
\left(p^{A, k, 1} D^{A, 1}(r, q)+p^{A, k, 2} D^{A, 2}(r, q)+p^{A, s i m, 1}\left(\frac{\alpha r a_{1}}{D}\right)+p^{A, s i m, 2}\left(\frac{\alpha r a_{2}}{D}\right)\right) \\
+(1-\varphi)\left(D^{B, 1}\left(p^{B, k, 1}\right)+D^{B, 2}\left(p^{B, k, 2}\right)\right)(1-\varphi) p^{A, k, 1}
\end{array}\right]+ \\
& \lambda_{2} q\left[D^{A, 1}(r, q)+D^{A, 2}(r, q)+D^{B, 1}\left(p^{B, k, 1}\right)+D^{B, 2}\left(p^{B, k, 2}\right)\right]+\lambda_{3} r r_{0}\left(a_{1}+a_{2}\right)
\end{aligned}
$$

In Eq. (8), the first term indicates the taxes revenue from operator $A$. The second term states government satisfaction with quality. The third term refers to government satisfaction with coverage development rate.

\section{Numerical Evaluation}

In this section, a numerical example based on case study with a sensitivity analysis is presented to illustrate the above theoretical results and to gain some managerial insights.

\subsection{Numerical Examples}

In this section, inspired by the case study, the numerical examples are designed to illustrate theoretical results of coverage development rate contract of cellular networks. To conduct numerical evaluations, some of the parameters are assumed to be constant in all experiments as $\quad a_{1}=100000, a_{2}=50000, D=7000, b=500, k=60, m=14000000, p^{A, s i m, 1}=$ $20000, p^{A, s i m, 2}=150000, p^{B, \operatorname{sim}, 1}=20000, p^{B, \operatorname{sim}, 2}=60000, \lambda_{1}=0.75, \lambda_{2}=1, \lambda_{3}=1$.

The rest of the parameter values for all five examples are given in Table 2 .

Table 2. Parameter values for the numerical experiments

\begin{tabular}{lllllllll}
\hline No. & $c$ & $\varphi$ & $d$ & $r_{0}$ & $\alpha$ & $p^{A, k, 1}$ & $p^{A, k, 2}$ & $\eta$ \\
\hline 1 & 0.1 & 0.38 & 0.2 & 0.44 & 0.3 & 65 & 59 & 4.3 \\
2 & 0.1 & 0.4 & 0.21 & 0.44 & 0.32 & 63 & 57 & 4.4 \\
3 & 0.11 & 0.3 & 0.29 & 0.44 & 0.4 & 67 & 60 & 2.75 \\
4 & 0.1 & 0.3 & 0.3 & 0.4 & 0.4 & 67 & 57 & 4 \\
5 & 0.13 & 0.29 & 0.28 & 0.43 & 0.45 & 66 & 61 & 3.5 \\
\hline
\end{tabular}

In Example \#1, in the decentralized model, when the first principal minors for operator A and operator $\mathrm{B}$ are negative $\left(-2(1-\varphi) m r_{0}{ }^{2}=-3686144<0,-2 b=-1000<0\right)$ and the second principal minors for them are positive $\left(4(1-\varphi)^{2} m k r_{0}{ }^{2}=300789350.4>0,4 b^{2}=\right.$ $4000000>0$ ), the optimal coverage development rate of operator $A$ is placed at 1.012 and 
the optimal quality level of the operator $A$ is 0.52 . The optimal selling price of the prepaid and permanent SIM cards' services for operator $B$ are placed at 74.75 and 62.30 . The optimal profits for the operator $A$ and the operator $B$ in the decentralized model are $\Pi_{A}=778998.370$ and $\Pi_{B}=1.381 \times 10^{6}$ thousand tomans ${ }^{2}$. After the implementation of the coordination plan, the optimal coverage development rate of operator $A$ is placed at 2.07 and the optimal quality level of the operator $A$ is 1.06. The optimal selling price of the prepaid and permanent SIM cards' services for operator B are placed at 107.41 and 78.63. After contract, the optimal profit for operator $A$ increases to $\Pi_{A}=1.457 \times 10^{6}$ and the optimal profit for operator $B$ decreases to $\Pi_{B}=1.146 \times 10^{6}$ thousand tomans. The optimal utility of government is $1.955 \times 10^{6}$ and tax rate coefficient is 0.391 . The results of the model indicate that after the contract between government and operators, operator $A$ increases its coverage development rate and quality level; with increasing this factors the demand of operator $A$ increases and subsequently the profit of operator $A$ increases. On the other hand, operator $\mathrm{B}$ gains less profit than the decentralized system, due to the lack of government support and participation in infrastructure cost, sustain high costs. In order to counteract the decline in profits, operator $B$ increases its sales prices of the prepaid and permanent SIM cards' services. Tables 2,3 , and 4 summarize the parameter values related to the five presented numerical examples.

Table 3. The results of the numerical examples before/after coordination plan

\begin{tabular}{llllllll}
\hline \multirow{2}{*}{ No. } & $\begin{array}{l}\text { Before/After } \\
\text { contract }\end{array}$ & $\begin{array}{l}\text { Operator } A^{\prime} \text { 's } \\
\text { profit }\end{array}$ & $\begin{array}{l}\text { Operator } B \text { s } \\
\text { profit }\end{array}$ & $\begin{array}{l}\text { Development } \\
\text { rate }\end{array}$ & $\begin{array}{l}\text { Quality } \\
\text { level }\end{array}$ & $\begin{array}{l}\text { Permanent } \\
\text { SIM's price }\end{array}$ & $\begin{array}{l}\text { prepaid } \\
\text { SIM's price }\end{array}$ \\
\hline 1 & Before & 778998.370 & $1.381 \times 10^{6}$ & 1.012 & 0.52 & 62.30 & 74.75 \\
& After & $1.457 \times 10^{6}$ & $1.146 \times 10^{6}$ & 2.07 & 1.06 & 78.63 & 107.41 \\
2 & Before & 726589.700 & $1.274 \times 10^{6}$ & 1.017 & 0.54 & 59.77 & 71.86 \\
& After & $1.640 \times 10^{6}$ & 423105.876 & 2.32 & 1.22 & 79.31 & 110.93 \\
3 & Before & $1.413 \times 10^{6}$ & $1.039 \times 10^{6}$ & 1.05 & 0.73 & 65.94 & 76.30 \\
& After & $1.585 \times 10^{6}$ & 891746.59 & 1.76 & 1.22 & 75.29 & 95.01 \\
4 & Before & $1.406 \times 10^{6}$ & 941261.13 & 1.13 & 0.72 & 63.28 & 75.79 \\
& After & $1.467 \times 10^{6}$ & 819005.39 & 1.82 & 1.15 & 71.57 & 92.38 \\
5 & Before & $1.452 \times 10^{6}$ & 971332. & 1.08 & 0.68 & 67.36 & 73.83 \\
& After & $1.567 \times 10^{6}$ & 716523. & 1.77 & 1.11 & 75.48 & 90.07 \\
\hline
\end{tabular}

Table 4. The results of the numerical examples for government

\begin{tabular}{lll}
\hline No. & $\gamma$ & Government's profit \\
\hline 1 & 0.391 & $1.955 \times 10^{6}$ \\
2 & 0.386 & $2.206 \times 10^{6}$ \\
3 & 0.416 & $2.00 \times 10^{6}$ \\
4 & 0.409 & $1.872 \times 10^{6}$ \\
5 & 0.414 & $2.00 \times 10^{6}$ \\
\hline
\end{tabular}

\footnotetext{
${ }^{2}$ Iranian toman is equal to 10 Iranian rials (IRR)
} 


\subsection{Sensitivity Analysis}

Some parameters have significant impacts on the performance of cellular networks and the results of the contract system. First, the impact of $\varphi$ on the profits of operator $A$, operator $B$, and government are investigated and the results are shown in Fig. 2. As can be seen in Fig. 2, $\varphi$ has a positive effect on the profits of operator $A$ and the government; on the other hand, on the profit of operator $B$ applies a two-part effect, in the first part, more profitable and in the second part reducing profits. As Participation rate $\varphi$ increases, the participation of operator $A$ and the government decreases in the cost of building a cellular network. As a result, with the increase in rate $\varphi$, the profit of operator $A$ and the government increases and the profit of operator $B$ decreases. However, it is important to note that operator $B$ purchases a part of its demand from operator $A$ with a discount based on this rate, thus, the profit of operator $B$ first increases and then decreases with increasing $\varphi$. If operator $B$ contributes more than 0.4 in the costs, then it will lose a lot, so it will not be more involved in any circumstances.

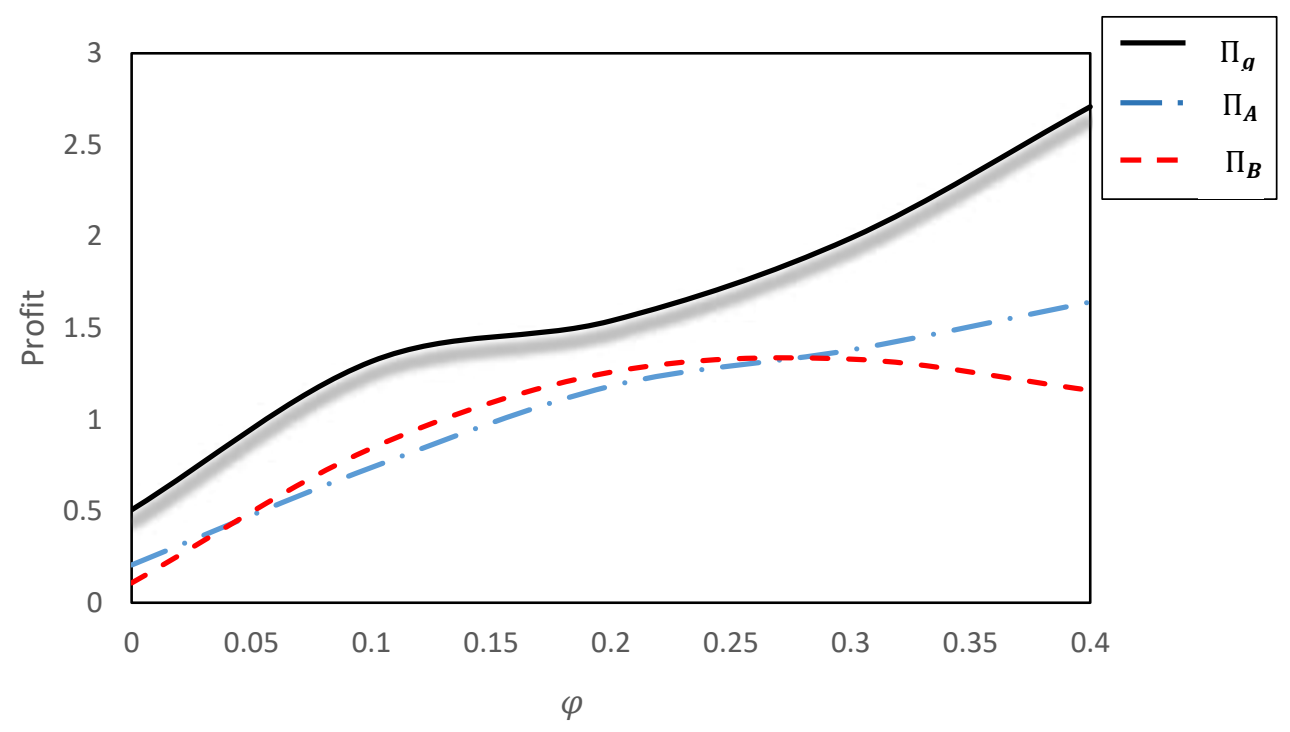

Fig. 2. Alteration of $\varphi$ vs. Beneficiary's profit function.

The coefficient effects of coverage development rate $r_{0}$ on network members' profits are also investigated and the results are shown in Figs. 3. As illustrated in Figs. 3, $r_{0}$ has a positive effect on operator $A$ 's profit because as $r_{0}$ increases the coverage rate increases and consequently the demand of operator $A$ increases; on the other hand, the government participation in costs increases and as a result, operator $A$ 's profit increases. The effect of this parameter on the profit of the operator $B$ is twofold, it initially profit increases with increasing $\varphi$, and then decreases. By increasing this parameter, demand increases and benefits the operator $B$, but due to increased coverage, the cost of constructing the developed segment rises and reduces operator $B$ 's profits. The government's profit decreases and then 
increases with this parameter' increase, because as the rate of coverage increases, government subsidy and cost participation will increase, but by increasing demand and gaining profit with operators, the government tax increases, which makes it profitable.

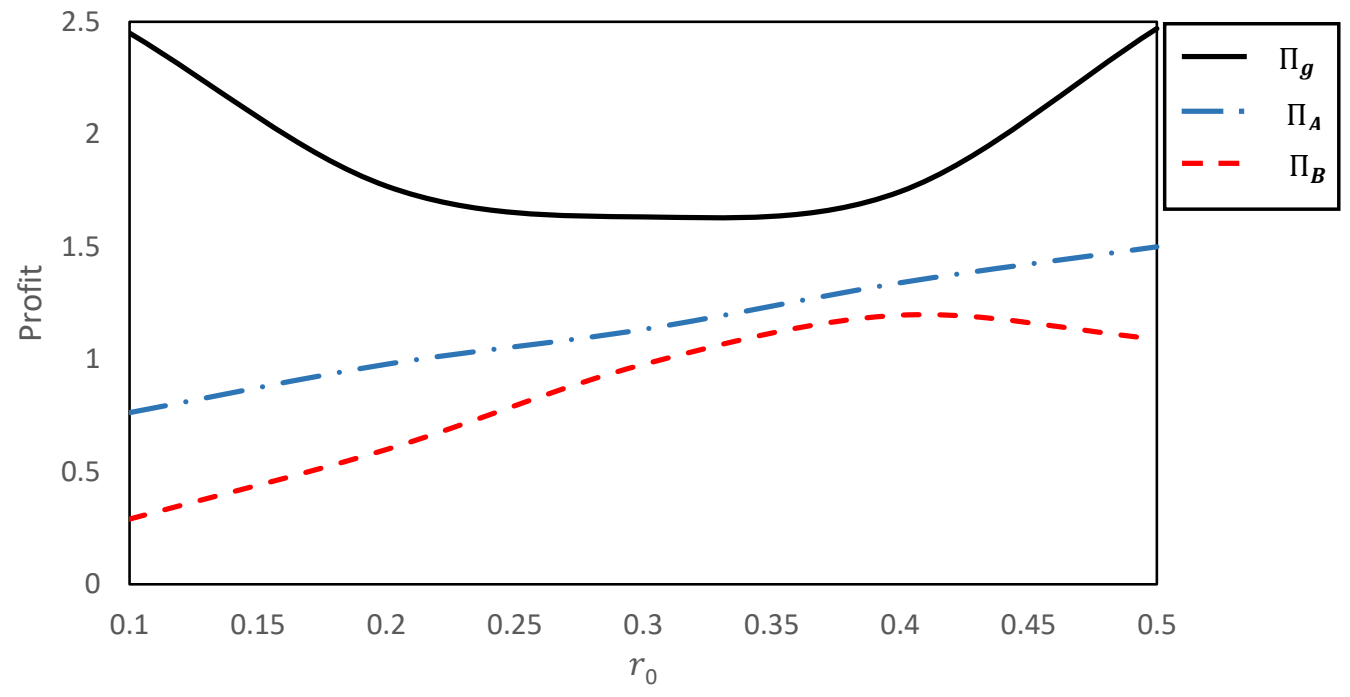

Fig. 3. Alteration of $r_{0}$ vs. Beneficiary's profit function.

The effect of $\alpha$ on cellular network members is investigated in Fig. 4, which indicates that $\alpha$ has an adverse effect on operator $B$ s profit. On the other side, $\alpha$ has a positive effect on operator $A$ 's and government's profit. As $\alpha$ increases, the demand of operator $A$ increases and the demand of operator $B$ decreases. Consequently the profit of operator $A$ increases and profit of operator $B$ decreases; as a result, the income from government tax increases and the government's profit rises.

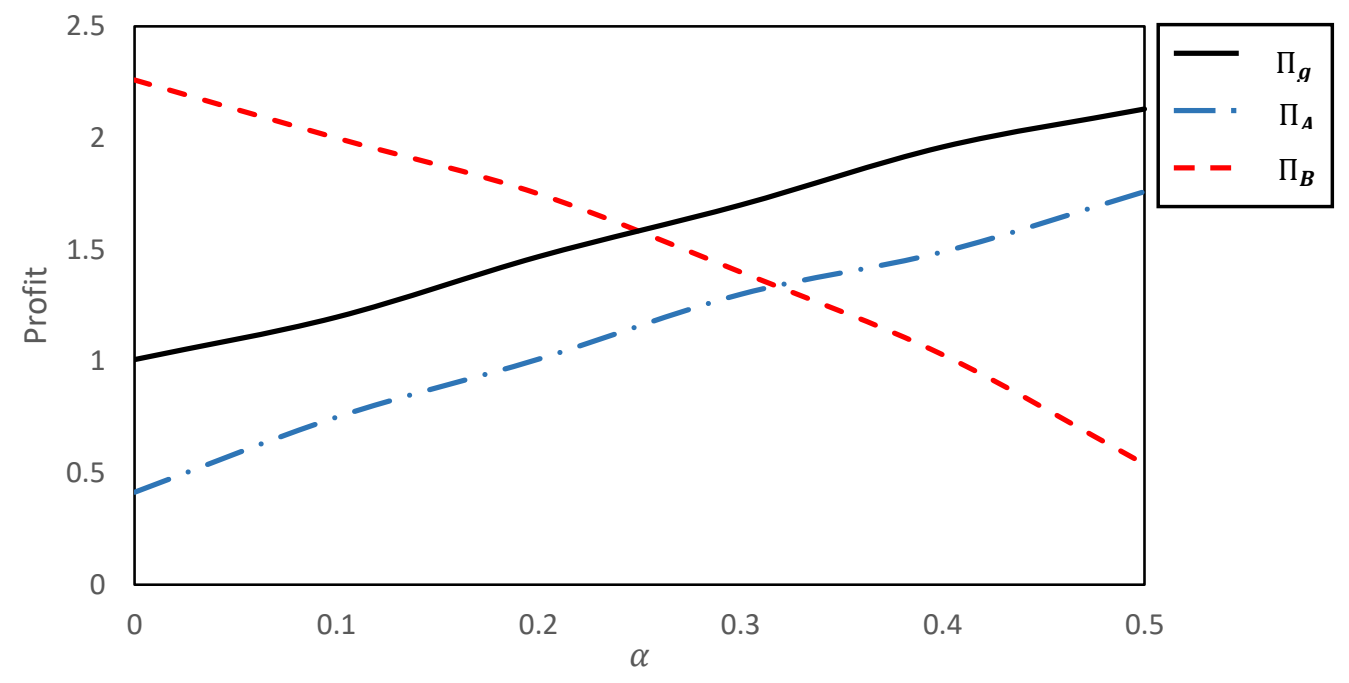

Fig.4. Alteration of $\alpha$ vs. Beneficiary's profit function. 
The impact of government tax coefficient is also investigated in Fig. 5. The behavior of the government's utility function with the tax coefficient is a dual behavior, initially as $\gamma$ increases the government's utility increase and reaching 0.4 in its own afterward; after that as $\gamma$ increases, the government's utility decreases. Due to increasing $\gamma$, the taxes received from operator $A$ increases, so that the operator $A$ cannot increase the quality and coverage; thus customer satisfaction decreases and consequently the government utility decreases. Therefore, the government should keep $\gamma$ between 0.3 and 0.4 , in order to both sides have optimal profit.

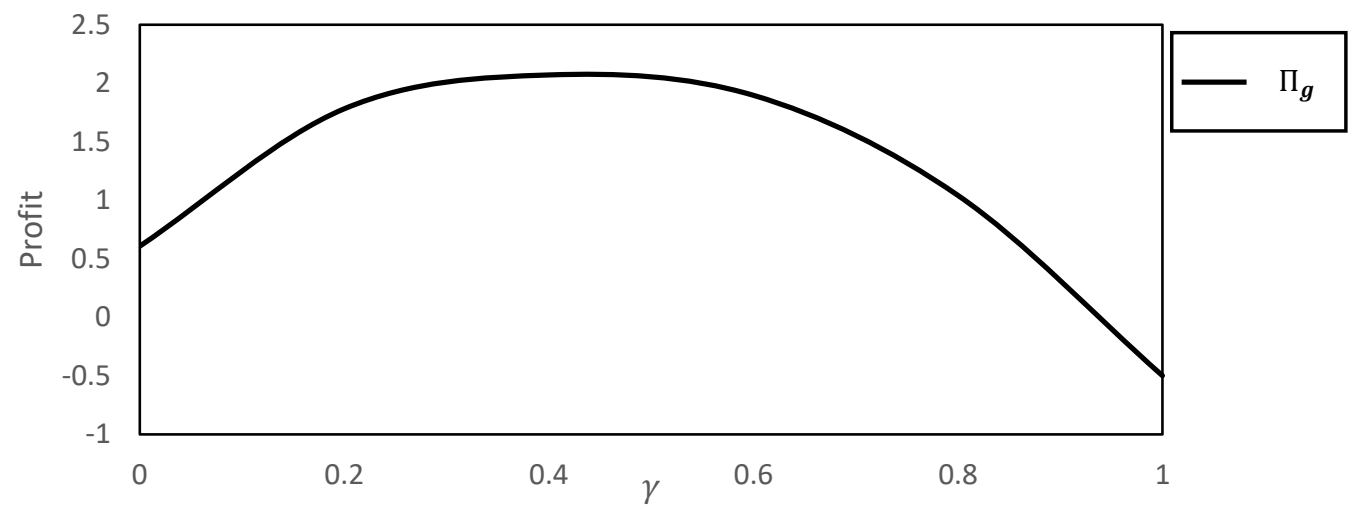

Fig.5. Alteration of $\gamma$ vs. Beneficiary's profit function.

Finally, the effect of $c$ is investigated in Fig. 6. As can be seen in Fig. 6, $c$ has a positive effect operator B's profit and has a negative effect on operator $A$ and government's profit. As $c$ increases, because of the sale prices of operator A's SIM cards are more than operator $B$ s SIM cards, operator $B$ attracts more demand and its profit increases; conversely, the demand of operator $A$ decreases and its profit decreases. On the other hand, the government' profit is related to operator $A$ 's profit; consequently when the profit of operator $A$ decreases, the government' profit decreases.

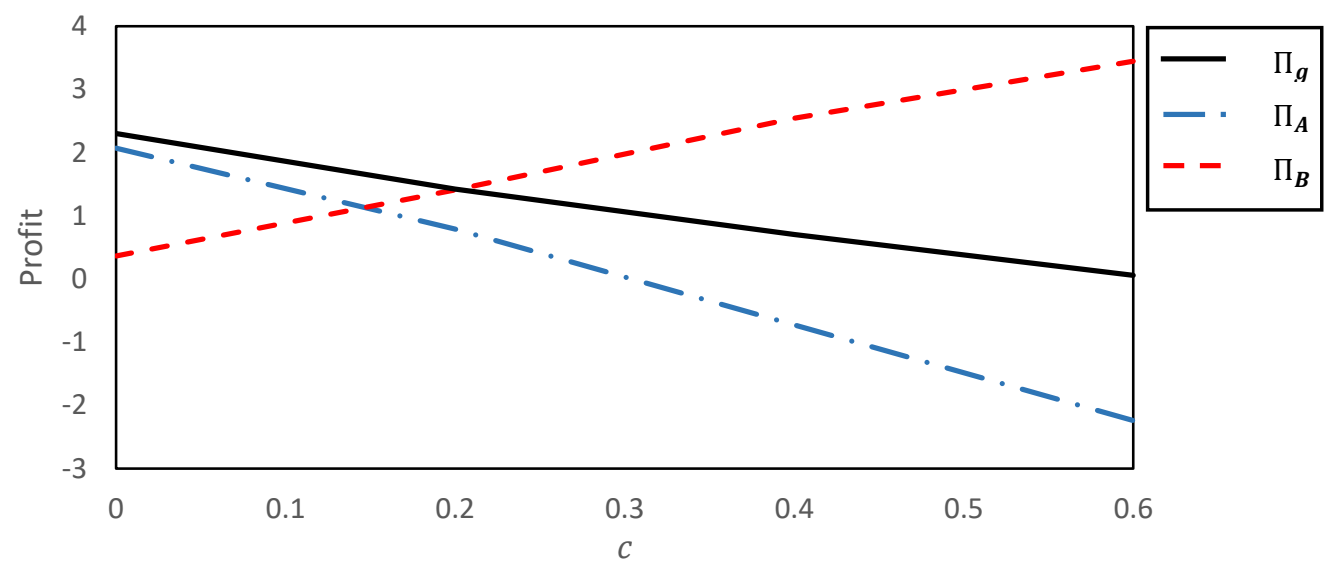

Fig.6. Alteration of $c$ vs. Beneficiary's profit function. 


\section{Conclusions}

In recent years, mobile smartphones have become an integral part of people's lives. For this reason, there is a space suitable for the business of mobile operators, each offering better and cheaper services to attract more demand. In the competitive environment between mobile operators, the service level includes the quality level of service provided and the coverage level as a competitive advantage. Sometimes, due to the remote and fragile nature of some of the areas, operators are working collaboratively in order to building antennas to serve customers. In this study, we considered a two old and new cellular operators under the influence of government intervention in one area. Because of the high cost of infrastructure and with the aim of responding to customer demand, the new operator opted to participate in infrastructure costs of the old operator. The new operator in the competitive situation in the area decides to price the sales price of SIM cards and services. The old operator aims to increase its profits by seeking to raise the quality level of service and coverage development rate in the region, in order to be able to respond to more requests. On the other hand, the government considers incentive schemes to support mobile operators. The government plan is that to take part in the infrastructure cost of the old operator and receive an income tax, and to support the new operator, declare it exempt from tax. The government subsidy contract with the old operator is based on the coverage level of the antenna and supports the operator to increase the coverage level. Hence, after the contract between government and operators, operator $\mathrm{A}$ increases its coverage development rate and quality level; with increasing this factors the demand of operator $A$ increases and subsequently the profit of operator $A$ increases. On the other hand, operator $B$ gains less profit than decentralized system, due to the lack of government support and participation in infrastructure cost, sustain high costs. In order to counteract the decline in profits, operator $B$ increases its sales prices of the prepaid and permanent SIM cards' services.

Further studies may be extended to consider the energy parameter as one of the decision variables of operators to reduce costs. Also, to reduce environmental pollution, laws such as cap and trade can be implemented to reduce carbon emissions. On the other hand, the relationship between the two partners can be linked to a competitive and commercial relationship such as the purchase and sale of antennas and services, and contracts are being made to coordinate these two operators. In addition, due to the efforts of operators to reduce energy costs, energy can be considered as a decision variable. 


\section{Compliance with ethical standards}

Conflict of interest: The authors declare that they have no known competing financial interests or personal relationships that could have appeared to influence the work reported in this paper.

Informed consent: Consent to submit has been received explicitly from all co-authors, as well from the responsible authorities-tacitly or explicitly-at the institute/organization where the work has been carried out, before the work is submitted.

Ethical approval: This article does not contain any studies with human participants or animals performed by any of the authors.

\section{References}

Berrocal-Plaza, V., Vega-Rodríguez, M. A., \& Sánchez-Pérez, J. M. (2014). On the use of multiobjective optimization for solving the location areas strategy with different paging procedures in a realistic mobile network. Applied Soft Computing, 18, 146-157.

Berrocal-Plaza, V., Vega-Rodríguez, M. A., \& Sánchez-Pérez, J. M. (2015). Optimizing the mobility management task in networks of four world capital cities. Journal of Network and Computer Applications, 51, 18-28.

Chan, A. and McNaught, K.R., 2008. Using Bayesian networks to improve fault diagnosis during manufacturing tests of mobile telephone infrastructure. Journal of the Operational Research Society, 59(4), pp.423-430.

Chang, J.-H., \& Tassiulas, L. (2004). Maximum lifetime routing in wireless sensor networks. IEEE/ACM Transactions on networking, 12(4), 609-619.

Cheng, X., Narahari, B., Simha, R., Cheng, M. X., \& Liu, D. (2003). Strong minimum energy topology in wireless sensor networks: NP-completeness and heuristics. IEEE transactions on mobile computing, 2(3), 248-256.

Dahi, Z. A., Alba, E., \& Draa, A. (2018). A stop-and-start adaptive cellular genetic algorithm for mobility management of GSM-LTE cellular network users. Expert Systems with Applications, 106, 290-304.

Ekmen, M., \& Altın-Kayhan, A. (2017). Reliable and energy efficient wireless sensor network design via conditional multi-copying for multiple central nodes. Computer Networks, 126, 57-68.

Fander, A., \& Yaghoubi, S. (2021a). Impact of fuel-efficient technology on automotive and fuel supply chain under government intervention: A case study. Applied Mathematical Modelling, 97, 771-802.

Fander, A., \& Yaghoubi, S. (2021b). Mathematical models for mobile network members' coordination through coverage development-based contract. Flexible Services and Manufacturing Journal, (Accepted, In press).

Hafezalkotob, A. (2015). Competition of two green and regular supply chains under environmental protection and revenue seeking policies of government. Computers \& Industrial Engineering, 82, 103-114.

Hafezalkotob, A. (2018). Direct and indirect intervention schemas of government in the competition between green and non-green supply chains. Journal of Cleaner Production, 170, 753-772.

Ji, Z., \& Liu, K. R. (2007). Cognitive radios for dynamic spectrum access-dynamic spectrum sharing: A game theoretical overview. IEEE Communications Magazine, 45(5).

Kamal, H., Coupechoux, M., \& Godlewski, P. (2009). Inter-operator spectrum sharing for cellular networks using game theory. Paper presented at the Personal, Indoor and Mobile Radio Communications, 2009 IEEE 20th International Symposium on.

Keskin, M. E. (2017). A column generation heuristic for optimal wireless sensor network design with mobile sinks. European Journal of Operational Research, 260(1), 291-304.

Kharaji Manouchehrabadi, M., \& Yaghoubi, S. (2020). A game theoretic incentive model for closed-loop solar cell supply chain by considering government role. Energy Sources, Part A: Recovery, Utilization, and Environmental Effects, 1-25. DOI: 10.1080/15567036.2020.1764150 
Lanza-Gutierrez, J. M., \& Gomez-Pulido, J. A. (2015). Assuming multiobjective metaheuristics to solve a threeobjective optimisation problem for Relay Node deployment in Wireless Sensor Networks. Applied Soft Computing, 30, 675-687.

Liu, L., Hu, B., \& Li, L. (2010). Energy conservation algorithms for maintaining coverage and connectivity in wireless sensor networks. IET communications, 4(7), 786-800.

Mahmoudi, R., \& Rasti-Barzoki, M. (2018). Sustainable supply chains under government intervention with a realworld case study: An evolutionary game theoretic approach. Computers \& Industrial Engineering, 116, 130143.

Mohamadi, A., Yaghoubi, S. and Pishvaee, M.S. (2016). Fuzzy multi-objective stochastic programming model for disaster relief logistics considering telecommunication infrastructures: a case study. Operational Research, pp.1-41.DOI: $10.1007 / \mathrm{s} 12351-016-0285-2$

Qin, X., Wang, X., Wang, L., Lin, Y., \& Wang, X. (2019). An Efficient Probabilistic Routing Scheme based on Game Theory in Opportunistic Networks. Computer Networks 149 (2019): 144-153.

Salih, Y. K., Hang See, O., Ibrahim, R. W., Yussof, S., \& Iqbal, A. (2015). A novel noncooperative game competing model using generalized simple additive weighting method to perform network selection in heterogeneous wireless networks. International Journal of Communication Systems, 28(6), 1112-1125.

Sheu, J.-B., \& Chen, Y. J. (2012). Impact of government financial intervention on competition among green supply chains. International Journal of Production Economics, 138(1), 201-213.

Srivastava, V., Neel, J. O., MacKenzie, A. B., Menon, R., DaSilva, L. A., Hicks, J. E., . . Gilles, R. P. (2005). Using game theory to analyze wireless ad hoc networks. IEEE Communications Surveys and Tutorials, 7(1-4), 4656.

Talbi, E.-G., Cahon, S., \& Melab, N. (2007). Designing cellular networks using a parallel hybrid metaheuristic on the computational grid. Computer Communications, 30(4), 698-713.

Trestian, R., Ormond, O., \& Muntean, G.-M. (2012). Game theory-based network selection: Solutions and challenges. IEEE Communications surveys \& tutorials, 14(4), 1212-1231.

Xifilidis, T., \& Psannis, K. E. (2018). Caching hit probability and Compressive Sensing perspective for mobile cellular networks. Simulation Modelling Practice and Theory, 87, 92-98.

Zand, F., Yaghoubi, S., Sadjadi, S.J., (2019). Impacts of government direct limitation on pricing, greening activities and recycling management in an online to offline closed loop supply chain. Journal of Cleaner Production, 215, 1327-1340.

Zhang, H., \& Hou, J. C. (2005). Maintaining sensing coverage and connectivity in large sensor networks. Ad Hoc \& Sensor Wireless Networks, 1(1-2), 89-124. 